\title{
SHARP BOUNDS FOR TOADER MEAN IN TERMS OF CONTRAHARMONIC MEAN WITH APPLICATIONS
}

\author{
Yu-Ming CHU, MiAO-Kun WANG AND XiAO-YAN MA
}

Abstract. We find the greatest value $\lambda$ and the least value $\mu$ in $(1 / 2,1)$ such that the double inequality $C(\lambda a+(1-\lambda) b, \lambda b+(1-\lambda) a)<T(a, b)<C(\mu a+(1-\mu) b, \mu b+(1-\mu) a)$ holds for all $a, b>0$ with $a \neq b$, and give new bounds for the perimeter of an ellipse. Here, $T(a, b)=\frac{2}{\pi} \int_{0}^{\pi / 2} \sqrt{a^{2} \cos ^{2} \theta+b^{2} \sin ^{2} \theta} d \theta$, and $C(a, b)=\left(a^{2}+b^{2}\right) /(a+b)$ denote the Toader, and contraharmonic means of two positive numbers $a$ and $b$, respectively.

Mathematics subject classification (2010): 26E60, 33E05.

Keywords and phrases: Toader mean, contraharmonic mean, complete elliptic integrals.

\section{REFERENCES}

[1] G. Almkvist And B. Berndt, Gauss, Landen, Ramanujan, the arithmetic-geometric mean, ellipses, $\pi$, and the Ladies diary, Amer. Math. Monthly 95 (1988), 585-608.

[2] H. ALZER AND S.-L. QIU, Monotonicity theorems and inequalities for the complete elliptic integrals, J. Comput. Appl. Math. 172, 2 (2004), 289-312.

[3] G. D. Anderson, M. K. Vamanamurthy and M. Vuorinen, Conformal Invariants, Inequalities, and Quasiconformal Maps, John Wiley \& Sons, New York, 1997.

[4] R. W. Barnard, K. Pearce And K. C. Richards, A monotonicity property involving ${ }_{3} F_{2}$ and comparisons of the classical approximations of elliptical arc length, SIAM J. Math. Anal. 32, 2 (2000), 403-419.

[5] J. M. Borwein And P. B. Borwein, Pi and the AGM, John Wiley \& Sons, New York, 1987.

[6] T. R. Chandrupatla AND T. J. Osler, The perimeter of an ellipse, Math. Sci. 35 (2010), 122-131.

[7] Y.-M. CHU AND S.-W. Hou, Sharp bounds for Seiffert mean in terms of contraharmonic mean, Abstr. Appl. Anal. (2012), Article ID 425175, 6 pages.

[8] Y.-M. ChU AND M.-K. WANG, Optimal Lehmer mean bounds for the Toader mean, Results Math. 61 (2012), 223-229.

[9] Y.-M. ChU, M.-K. WANG AND S.-L. QIU, Optimal combinations bounds of root-square and arithmetic means for Toader mean, Proc. Indian Acad. Sci. 122, 1 (2012), 41-51.

[10] Y.-M. ChU, M.-K. WANG, S.-L. QIU AND Y.-F. QIU, Sharp generalized Seiffert mean bounds for Toader mean, Abstr. Appl. Anal. (2011), Article ID 605259, 8 pages.

[11] G. TOADER, Some mean values related to the arithmetic-geometric mean, J. Math. Anal. Appl. 218, 2 (1998), 358-368.

[12] M. VUORINEN, Hypergeometric functions in geometric function theory, Special functions and differential equations (Madras, 1997), 119-126, Allied Publ., New Delhi, 1998.

[13] M.-K. WANG, Y.-M. ChU, S.-L. QIU AND Y.-P. JIANG, Bounds for the perimeter of an ellipse, J. Approx. Theory 164 (2012), 928-937. 